\title{
Entraves à l'attitude ludique avec un jeu sérieux intégré dans une formation managériale : un exercice plus qu'un jeu?
}

\section{Lydia Martin}

\section{(2) OpenEdition}

\section{Journals}

Édition électronique

URL : http://journals.openedition.org/sdj/796

DOI : $10.4000 /$ sdj.796

ISSN : 2269-2657

Éditeur

Laboratoire EXPERICE - Centre de Recherche Interuniversitaire Expérience Ressources Culturelles Education

\section{Référence électronique}

Lydia Martin, «Entraves à l'attitude ludique avec un jeu sérieux intégré dans une formation

managériale : un exercice plus qu'un jeu ? », Sciences du jeu [En ligne], 7 | 2017, mis en ligne le 22 février 2017, consulté le 28 mars 2021. URL : http://journals.openedition.org/sdj/796 ; DOI : https:// doi.org/10.4000/sdj.796

\section{Ce document a été généré automatiquement le 28 mars 2021.}

\section{cc)}

La revue Sciences du jeu est mise à disposition selon les termes de la Licence Creative Commons Attribution - Pas d'Utilisation Commerciale - Pas de Modification 4.0 International. 


\title{
Entraves à l'attitude ludique avec un jeu sérieux intégré dans une formation managériale : un exercice plus qu'un jeu?
}

\author{
Lydia Martin
}

1 L'apprentissage par le jeu n'est pas nouveau. Dès le $\mathrm{XV}^{\mathrm{e}}$ siècle, avec le mouvement humaniste en Italie, on recense l'oxymore Serio Ludere que l'on pourrait traduire par «jouer sérieusement ». Ce terme renvoie à l'idée de traiter d'un sujet « sérieux » avec de «l'humour », avec une approche « amusante » (Alvarez \& Djaouti, 2010, p. 179).

2 Avec les jeux sérieux, l'apprentissage évoluerait vers l'assimilation de connaissances de façon amusante et facile. Cependant, l'apprentissage par le jeu est plus complexe. Il pose un certain nombre de questions qui, à ce jour, sont encore sans réponses, comme la rupture cognitive entre le jeu et le travail (Pomian, 2009). L'appellation même de jeu sérieux est au service, entre autres, d'un projet de formation et d'objectifs pédagogiques, ce qui met en tension la logique du jeu et la logique d'apprentissage (Brougère, 2012 ; Lavigne, 2014). Les représentations individuelles détermineraient la façon dont les individus perçoivent ce qui est de l'ordre du jeu et ce qui ne l'est pas. Il existe un glissement de l'analogie à l'identité des rapports entretenus entre « travail et jeu » : le jeu représentant la diversion, la joie, la fantaisie et la détente pouvant alléger les choses de la vie ; alors que le mot « sérieux » renvoie à la responsabilité, la réalité et les actions avec des conséquences (Marcano, 2008). De par cette tension entre le travail et le jeu, le serious game se situerait déjà à la marge du jeu.

Dans cet article, nous questionnons le sens même du jeu lorsque celui-ci est vécu comme obligatoire, dans un programme de formation dédié aux managers appartenant à la même organisation. L'impact que cela peut avoir sur la posture qu'adopte le manager avant de «jouer» est pris en compte pour penser ce qui entrave l'acte de jouer. Nous pouvons faire le lien avec l'attitude ludique inhérente au « jouer » centrale dans l'œuvre de Jacques Henriot (1983, p. 83) : 
pour jouer, il faut entrer dans le jeu. Pour entrer dans le jeu, il faut savoir que c'est un jeu. Il y a donc, de la part de celui qui se met à jouer, une compréhension préalable du sens du jeu. L'attitude ludique, comme toute attitude, se prend. Comme toute attitude, elle se comprend.

4 Après avoir présenté la méthodologie de la recherche, nous préciserons en quoi soumettre des managers à un dispositif de formation présenté comme un jeu sérieux peut aider à comprendre ce qui fait la frontière du jeu. Cette frontière serait la rencontre "entre des objectifs "sérieux" et des modalités ludiques, réunis dans un même objet qui crée, de ce fait, une nouvelle réalité hybride permettant la communication entre les deux mondes. Les jeux sérieux pourraient-ils être qualifiés d' "objets-frontière" ?» (Lavigne, 2016). Accepter ou pas de jouer dans un programme de formation managériale semble exclu, nous n'avons observé aucune situation de refus « explicité ». Le «joueur » pourrait en effet faire semblant de jouer tout en refusant mentalement l'entrée dans le jeu.

Comment parler de jeu sans jouer? Peut-on rester grave et compassé devant un sujet dont le moins qu'on puisse dire est qu'il prête à jouer, non seulement avec les mots que l'on emploie mais aussi et plus profondément avec le sens même d'une réalité fuyante, rebelle, ne se dévoilant et ne se laissant entrevoir que pour se dérober aussitôt? Qui parlerait de jeu sans jouer plus ou moins ne jouerait pas le jeu (Henriot, 1989, p. 10).

5 Notre travail de recherche nous a permis d'amorcer une réflexion autour des marges $\mathrm{du}$ jeu lorsque celui-ci devient une pratique prescrite dans des dispositifs de formations managériaux. Quels sont alors les empêchements au «jouer »? Certains empêchements avaient déjà été abordés lors d'une précédente publication : le sentiment d'être évalué, le bien faire, la culture d'entreprise ainsi que les processus inconscients dans les groupes (Martin, 2015).

6 Pour caractériser l'expérience des jeux vidéo l'étude de la structure ludique ne suffit pas (Triclot, 2011). On ne peut se contenter de regarder le game, la structure, sans prendre en compte le sujet jouant (Brougère, 2013). Nous proposons ici une autre approche en analysant deux études de cas très différentes dans la perception que les managers ont eue du dispositif de formation. Notre analyse nous permet de nous interroger sur les raisons de cette différence qui pourrait être due soit à l'intrusion du ludique dans le contexte professionnel soit de l'évaluation dans une activité a priori plutôt dévolue à la formation.

\section{Penser les marges du jeu dans les pratiques prescrites}

7 Le jeu est défini par Joan Huizinga (1988, p. 217) comme

une action qui se déroule dans certaines limites, de lieu, de temps et de volonté, dans un ordre apparent, suivant des règles librement consenties, et hors de la sphère de l'utilité et de la nécessité matérielles. L'ambiance du jeu est celle du ravissement et de l'enthousiasme, qu'il s'agisse d'un jeu sacré, ou d'une simple fête, d'un mystère ou d'un divertissement. L'action s'accompagne de sentiments et entraîne avec elle joie et détente.

8 Roger Caillois (1991) déduit de cette définition des caractéristiques fondamentales : le jeu est une activité libre; séparée (limites d'espace et de temps) ; incertaine ; improductive ; réglée et fictive. 
9 Nous retrouvons ici le play, qui est un jeu libre où, suivant les théories de Winnicott (1971) l'espace transitionnel permet de créer et de se "construire», ce play est différencié du game où l'espace est contraint. Le jeu contraint (game ou ludus dont nous parle Roger Caillois) n'offre aucun espace pour la créativité et ne permet pas de véritable travail de subjectivation. Le jeu « libre » (play ou paida) « prend la valeur d'une création ou d'une recréation de la subjectivité et produit une nouvelle forme de rapport à soi, à l'autre, au monde » (Roussillon, 2008 cité par Rinaudo, 2011, p. 81). L'espace du jeu est ainsi un espace transitionnel qui ouvre des possibles et où on peut agir sur ses propres modèles et représentations.

10 Jobert définit la représentation comme un

ensemble d'images mentales que nous nous construisons pour rendre compte des phénomènes de toute nature qui nous entourent et que nous rendons ainsi à la fois intelligibles, cohérents et maîtrisables lorsqu'il s'agit d'agir sur eux [...] ces représentations constituent une des voies de saisie du monde concret, un moyen individuel et collectif de façonner le réel (Jobert, 1986, p. 58).

11 Le jeu est un espace intermédiaire entre l'espace intérieur, le subjectif, et l'espace extérieur de la réalité. C'est un espace de développement où la créativité est prépondérante et favorise l'expérimentation dans un espace protégé où la sanction ne s'applique pas comme dans le réel. De plus, l'espace potentiel est l'espace du jeu, du « je », où ce qui est « trouvé » devient « créé ».

12 Le ludique ne serait pas que dans l'objet, mais dans le rapport que développe l'individu avec l'objet, c'est ce qu'Henriot nomme « l'attitude ludique » (1989, p. 10). Il s'agit d'une attitude adoptée qui participe à ce qu'on nomme couramment le jeu. L'une des conditions fondamentales en est le choix de jouer : on y entre de son plein gré et on peut en sortir quand on le désire.

13 Pour cela, Henriot (1969) définit trois caractéristiques au « jouer » comme attitude sur lesquels nous nous appuierons dans cet article :

- L'incertitude serait le caractère le plus apparent de toute conduite ludique (p. 82);

- La duplicité qui serait cette capacité à se «dédoubler », en mettant une certaine distance entre soi et le jeu (p. 82);

- L'illusion qui fait que le jeu peut se déployer (p. 83).

14 «Au plaisir que prend le joueur, on oppose la peine qu'éprouve le travailleur ; à la liberté du jeu, la contrainte du travail» (Henriot, 1989, p. 194). Le jeu ne serait-il pas avant tout un acte volontaire? Cela permet de s'interroger sur le jeu quand il passe "d'une pratique collective autonome en marge du travail prescrit à une pratique systématisée dans des dispositifs managériaux pour accroître la productivité et participer à l'évaluation de l'activité » (Le Lay, 2013, p. 2).

Jouer serait produire du jeu à partir des règles et les actualiser en les faisant siennes, en les exploitant individuellement et collectivement (Simon, 2005). C'est donc l'attitude que le joueur adopte qui l'amènera à « jouer » avec le système de règles, autrement dit avec la prescription, ou qui lui permettra de trouver des marges de manœuvre favorisant le déploiement du «jeu » et de l'espace intermédiaire dont parle Winnicott entre un dedans, subjectif, et un dehors, la réalité. Cependant, cette exploitation ne peut se faire que dans un environnement "suffisamment bon" pour reprendre le terme de Winnicott (1970, p. 56), c'est-à-dire à la fois « habitant » pour permettre cette exploitation et « résistant» avec un cadre contraint, fait de règles (Rinaudo, 2011). 
Dans le cadre d'un jeu sérieux, le formateur est garant de cet espace «suffisamment bon ».

La question du sérieux du jeu se pose dans le cas des jeux sérieux (Brougère, 2012 ; Rinaudo, 2011). L'imaginaire collectif associe souvent le jeu au loisir et l'oppose au travail. Les serious games distingueraient les jeux dits sérieux des autres qui ne seraient pas sérieux (Rinaudo, 2011). La définition du mot sérieux du Robert signifie : «qui ne peut prêter à rire ou être estimé sans conséquence » (Brougère, 2012). Le sérieux serait inhérent au jeu dont les conséquences sont à prendre au sérieux. Dans le sens anglophone, "serious » évoque une grande concentration : jouer sérieusement, ce qui peut vouloir dire jouer de manière concentrée. C'est pour cette raison que Julian Alvarez (2007) préfère traduire «serious » par « utilitaire » qui semble beaucoup plus proche de l'idée portée par cet oxymore. Alvarez, Libessart \& Haudegond (2014) s'appuient sur Etienne Armand Amato pour qui la fonction « utilitaire » s'entend dans le sens "productif», c'est-à-dire qui renvoie aux termes "transformation", " amélioration des compétences ", "adaptation au milieu » et «compréhension d'un phénomène». Une autre traduction de «serious» est aussi sévère, grave ou préoccupant. Ainsi Cortázar (2013) parlait de la « gravité du jeu ».

17 Nous nous intéresserons dans cet article au jeu vidéo non comme objet, mais comme expérience (Triclot, 2011) puisque la preuve du jeu ne peut être faite que de l'expérience même de la subjectivité : c'est-à-dire par l'analyse de l'expérience vécue (Henriot, 1969).

\section{Le terrain de la recherche}

\section{Un détournement d'usage}

18 Afin d'analyser la problématique des marges du jeu au sein de pratiques prescrites, nous nous appuyons sur une recherche qui s'est déroulée au sein du centre de formation d'un grand groupe industriel et qui a duré deux ans (2013 et 2014). Nous avons été confrontés à un détournement d'usage du simulateur d'hélicoptère EDITH utilisé par l'armée pour former les chefs de bord et les chefs de patrouille à la coopération intra et inter hélicoptère. Le centre de formation du groupe industriel a intégré ce simulateur le troisième jour d'une formation managériale de quatre jours intitulée "Team Management: du manager au leader" et l'a renommé SimLead (Simulateur de Leadership). SimLead est alors présenté comme un serious game aux managers le premier jour de la formation.

SimLead se déroule dans une salle sombre et tamisée où sont disposés deux hélicoptères par équipe. Dans chaque hélicoptère, trois managers, qui ne se côtoyaient pas avant cette formation, jouent un rôle (pilote, navigateur ou chef de bord), avec pour mission de sauver des rescapés suite à des inondations. Un jeu de rôle supplémentaire, celui du préfet dirigeant les opérations, est joué par le formateur, instructeur sur EDITH et ancien capitaine des armées. 
Figure 1

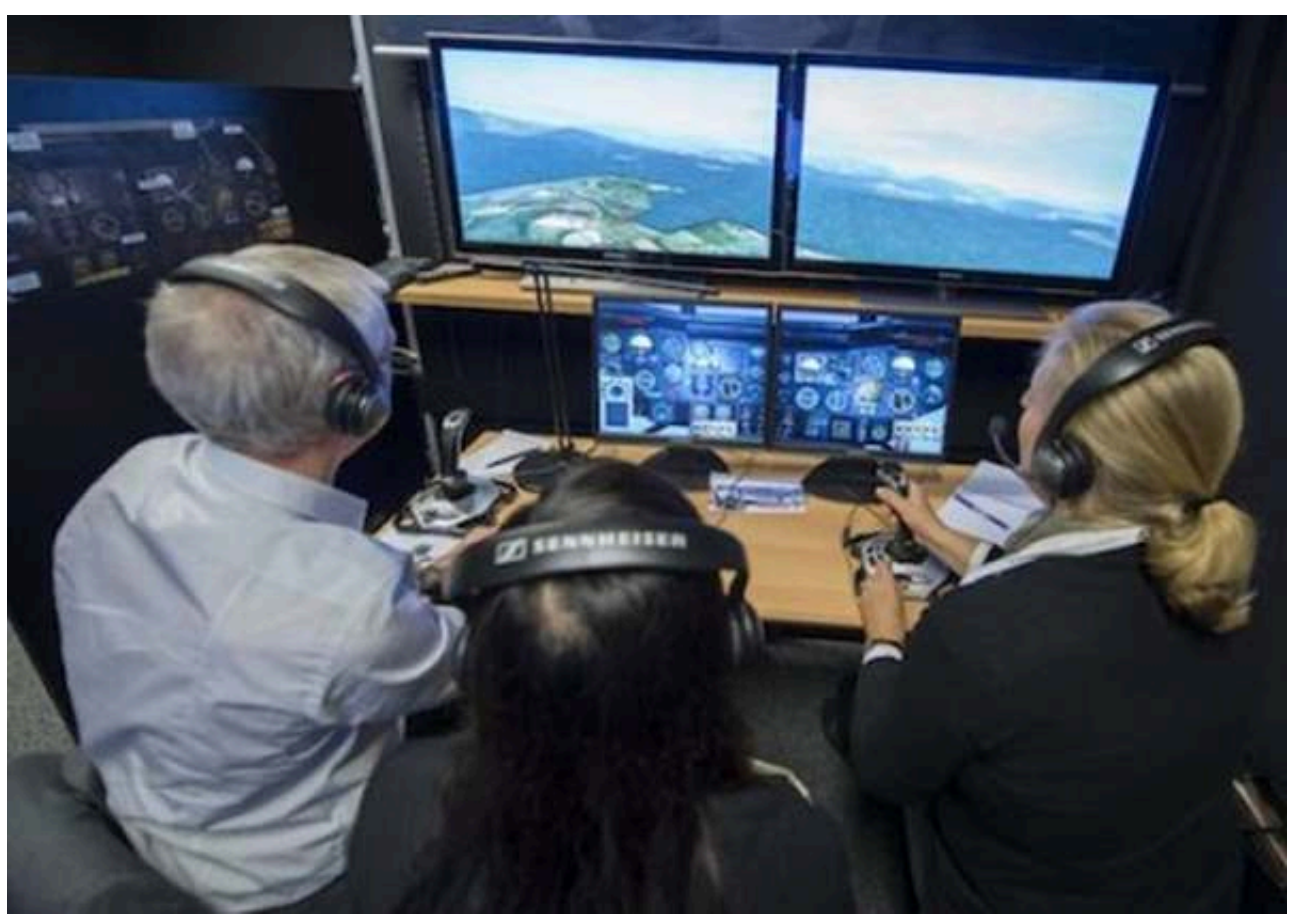

SimLead

20 Les objectifs pédagogiques sont : la constitution d'une équipe, la répartition des rôles et des responsabilités, la définition d'une stratégie, la gestion du risque et du stress, l'apprentissage organisationnel et la communication.

Les différents temps et exercices sont :

- Briefing et apprentissage au pilotage (le reste de l'équipe définit qui sauver en premier) : 45 minutes.

- $1^{\mathrm{er}}$ exercice « ramener le plus de monde » (carte différente pour chaque équipe) : 30-40 minutes.

- Préparation du $2^{\mathrm{e}}$ exercice : 5 à 15 minutes en fonction des groupes.

- $2^{\mathrm{e}}$ exercice « ramener les rescapés en fonction d'une valeur pécuniaire»dans un environnement compétitif (chaque équipe a la même carte) : 30-40 minutes.

- $3^{\mathrm{e}}$ exercice « ramener un groupe de VIP » dans un environnement compétitif (même zone de sauvetage pour les deux équipes) : 15-20 minutes.

Les exercices se déroulent dans un environnement "dynamique »: changements de directions, de lieux où déposer les rescapés, de réduction du temps alloué à la mission et de climat (neige, brouillard, etc.). Les concepteurs du centre de formation et d'une grande école de management ont été rencontrés et leur l'objectif était d'amener les équipes à se coordonner et à prendre des décisions en situation de crise. Sur l'ensemble de l'intervention, nous avons observé 16 sessions de SimLead sur une demi-journée chacune, soit 175 managers, et deux sessions de formation complètes ( 4 jours).

Le schéma ci-dessous représente le programme de la formation dans lequel est intégré SimLead : 
Figure 2

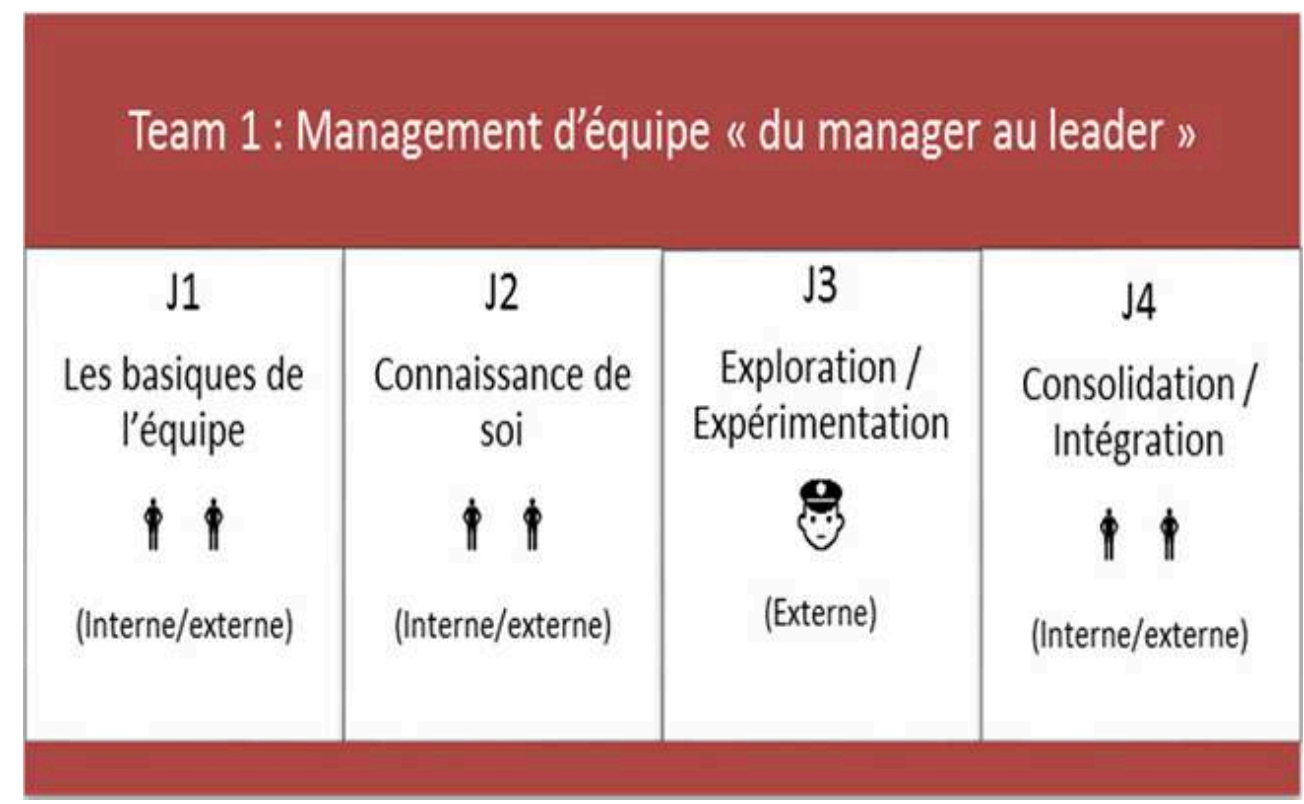

Programme complet de la formation

Le deuxième jour de la formation (« connaissance de soi »), les managers réalisent un questionnaire de personnalité: le MBTI (Myers-Briggs Type Indicator). Les deux premiers jours et le quatrième jour de la formation sont animés par un binôme de formateurs (un consultant interne et un coach externe) et le troisième jour, c'est un ancien capitaine de l'armée et instructeur sur EDITH qui anime SimLead.

\section{Jouet ou simulateur}

Lelardeux, Panzoli, Alvarez, Galaup \& Lagarrigue (2012) établissent un lien entre serious play et simulateur, mais démarquent les simulateurs des serious games. Ils qualifient de "serious play", les jeux sérieux qui se basent sur une structure play (jouet) et de "serious game" ceux qui se basent sur une structure game (jeu avec règles). Les deux peuvent avoir une fonction utilitaire. Le simulateur s'appuie sur des modèles numériques et reproduit un comportement fidèle de la réalité. La présence d'un formateur est indispensable pour animer et atteindre des objectifs dans une formation. Il manipule et adapte les scénarii en fonction des réactions des managers et des objectifs pédagogiques. Les travaux en ergonomie montrent que cette médiation n'est pas neutre et passive, elle oriente et guide la simulation.

26 A l'inverse, le jeu sérieux intègre un scénario pédagogique avec des objectifs, des contraintes et des moyens associés. Il s'affranchit ainsi de la présence d'une relation tierce. À l'instar du serious play, le serious game peut convoquer une métaphore, ce qui n'est pas le cas d'un simulateur. Dans les jeux sérieux, la métaphore est définie comme la substitution d'un univers de jeu par un contexte de référence qui favorise la fantaisie pour motiver et/ou immerger le joueur. Cet univers de substitution sera alors appelé un « univers métaphorique » (Lelardeux, Alvarez, Montaut, Galaup \& Lagarrigue, 2012).

Il nous semble que ce détournement d'usage pose principalement la question de la différence entre un jouet et un simulateur dont «le cadre, les personnes mises en présence et le contexte jouent très certainement des rôles clés dans la manière 
d'utiliser un même objet " (Alvarez, 2015, p. 5). Pour illustrer, cette distinction Julian Alvarez (2015) prend l'exemple de l'usage d'une poupée qui peut être un jouet ou un simulateur si elle est utilisée par des sages-femmes en formation. Ainsi, puisque la notion de contexte peut prédominer sur le dispositif, il convient de s'en détacher pour prendre en compte l'activité. Dans notre recherche, nous analysons le jeu comme une activité, comme un faire: «jouer c'est faire quelque chose. La chose que l'on fait présente deux versants: l'un objectif ou externe, l'autre subjectif ou interne" (Henriot, 1969 p. 47). Ce qui différencie des « faire » qui peuvent sembler identiques est le sens que le joueur leur donne (Brougère, 2013). Il s'agit bien de centrer l'analyse sur le jouer et le joueur.

\section{Analyse du jouer et des joueurs}

Nous avons rencontré 44 managers, tous volontaires, sur leur lieu de travail un mois après les sessions de formation. Seulement 7 d'entre eux se sont portés volontaires pour être rencontrés en amont de la formation pour des raisons de non-connaissance du dispositif. 35 des managers étaient des ingénieurs, les autres étaient dans des fonctions supports (achats, communication, ressources humaines, etc.). La population était très hétérogène : ils avaient entre 35 et 62 ans et une ancienneté de 2,5 à 33 ans au sein de l'entreprise.

Les matériaux ont été collectés suivant trois modalités : observations in situ pendant SimLead, entretiens individuels sur le lieu de travail avant et après l'expérience. Les observations des situations de SimLead ont été essentielles et se sont appuyées sur une grille d'observation concernant le travail en groupe, les interactions dans le groupe, le corps dans l'activité et les manifestations émotionnelles associées ainsi que les réseaux de communication. Dans l'observation, nous avons aussi écouté : les conversations, le contenu des échanges ou des discussions, les interpellations, les rires, etc. Nos observations nous ont permis de relever, en situation de jeu, des verbatim, les interactions dans l'équipe et entre les équipes, mais aussi les situations de repli et les consignes du formateur. Pour compléter nos observations, nous avons choisi la méthode des entretiens semi-directifs. La confidentialité des entretiens, la garantie de l'anonymat ainsi que l'autorisation demandée d'enregistrer ont permis l'instauration d'un climat de confiance.

En amont de la formation, les entretiens ont duré une heure. Notre grille d'entretiens préétablie, mais évolutive en fonction des rencontres, nous a permis de questionner sur les trajectoires professionnelles ; l'activité du manager ; la représentation qu'ils ont de leur métier et les attentes vis-à-vis de la formation. Un à deux mois après la formation et l'expérience de SimLead, les entretiens d'une durée de $1 \mathrm{~h} 30$ à 2 heures ont porté sur l'expérience elle-même, individuellement et collectivement, son vécu et les transformations éventuelles provoquées de retour au travail. Des liens ont été réalisés avec les grilles d'observation et les entretiens réalisés en amont.

31 Nous avons rencontré à nouveau dix managers afin de compléter les données de certains entretiens. Ces nouveaux entretiens ont permis d'approfondir individuellement la réflexivité déjà amorcée en groupe lors du débriefing, souvent jugé trop court par les managers, entre le vécu du jeu et les situations de travail réel mais.

Nous précisons que cette formation concerne 350 managers par an et qu'elle est un lieu propice à la diffusion des messages stratégiques. La présence des formateurs lors du 
déroulement de SimLead a été jugée coûteuse, et c'est la même raison qui a amené les responsables de la formation à ne pas solliciter la présence du formateur qui anime SimLead lors du débriefing le lendemain de l'expérience.

Sur les 44 managers rencontrés, nous avons observé et entendu deux vécus opposés ce qui explique le choix que nous faisons pour cet article de les analyser.

Les deux études de cas sélectionnées sont:

- François, 38 ans, Ingénieur et Responsable R\&D. Joueur dans la vie, il connait et comprend l'illusion d'un jeu, l'incertitude, et nous reviendrons sur son approche de la duplicité. Il a développé une attitude ludique en négociant certaines règles par exemple le changement de place des deux pilotes, changement observé une seule fois sur les 16 sessions. Nous avons réalisé trois entretiens avec lui, dont un en amont de la formation et un sous forme de récit de vie.

- Martine, 45 ans, Responsable finance. Elle n'a pas vécu l'outil comme un jeu, mais comme un exercice et est restée soumise aux systèmes de règles. Nous l'avons rencontrée à deux reprises après la formation.

Nous partirons de leur vécu afin de comprendre les conditions favorables chez le sujet et dans son environnement pour développer une attitude ludique, mais aussi les empêchements à son développement.

\section{Transfert d'une activité de jeu dans le travail}

\section{Une distance nécessaire entre soi et le jeu}

L'analyse de l'entretien avec François fait ressortir l'idée d'un échange entre les sphères de vie et le travail comme un sous-système dépendant du système de vie global de l'individu (Curie \& Hajjar, 1987 ; Curie \& Dupuy, 1994 ; Almudever, 2007). François transfère une activité de jeu dans l'activité professionnelle ce qui lui permet d'appréhender la structure de jeu avec une certaine distance.

Moi je suis un gros joueur par ailleurs donc je n'ai pas du tout le côté étonné par le système. Déjà de métier je fais de la simulation donc c'est un truc que je connais bien et à titre personnel je joue beaucoup et sur des jeux de plateau, des jeux de rôle, des jeux vidéo donc ce n'est pas un univers dans lequel j'aurais été extrêmement fasciné par défaut.

37 François distingue le Moi en deux instances: le «joueur» et la personne, ici le manager. Cette duplicité inhérente à l'attitude ludique permet de dissocier ce qui se passe entre le virtuel et le réel. Il nous explique :

[...] dans des jeux où c'est en plus un jeu sérieux, ce n'est pas si évident que les gens se disent ce n'est qu'un jeu, il n'y a pas d'enjeux, et que si quelqu'un dit: « tu n'as pas fait ce qu'il fallait», c'est un reproche au joueur, mais pas à la personne, certains vont dire " pourquoi tu m'as fait ça on n'est plus copain ", ceux qui ne font pas bien la différence entre jeu et réalité, ils restent dans le game et n'ont pas compris qu'ils n'étaient pas dans la réalité. Quand on est habitué à jouer, il est plus facile de faire ce glissement-là [...]

Pour faciliter ce glissement, nous introduisons la notion de « conséquences négociées sur la vie réelle» de Juul (2003) puisque tous les jeux sont potentiellement des outils d'apprentissage qui peuvent être utilisés dans la formation professionnelle. Ces conséquences seraient assignées et ces jeux à usage ludique dans le monde professionnel porteraient un degré de séparation du reste du monde puisque leurs 
conséquences seraient négociables. Cela impliquerait certainement que les règles du jeu soient explicitées au démarrage ainsi que les conséquences que le jeu peut avoir, ce qui n'est pas le cas à ce jour dans le dispositif étudié. Cela va donc bien plus loin que la "duplicité ». Ce sont aussi les interactions entre le monde du «jeu» et le monde « réel » qui permettent d'interpréter une activité comme une activité de jeu, c'est-àdire comme une activité de second degré (Brougère, 2005). Cette notion de second degré est un aspect essentiel dans le jeu, comme dans toutes les situations de «faire comme si ».

Mendel (1998) a intitulé une partie de son livre : « Le jeu avec le monde : sortir de soi ou pas » (pp. 438-440). Il aborde cette capacité à se centrer sur son environnement plus que sur soi et il explique qu'une des raisons à cet " auto-centrage " pourrait être une mère trop centrée sur l'enfant dans l'enfance. Ce perspective peut être utile pour amorcer une réflexion sur ce que François nomme : la « dissociation» moi/joueur. Ce « glissement », ce « dégagement » dont nous parle François pour «aller dans le play » n'est pas aisé.

[...] mine de rien, il y a un dégagement qui n'est pas si facile à obtenir pour aller dans le play puisque l'on est obligé, on ne peut pas dire : «non je n'y vais pas » et puis il y a une pression sociale : « ah pourquoi tu n'irais pas, tu as peur que l'on voie que tu n'es pas bon ".

Nous partons ici du postulat que sans cette dissociation du moi en tant que joueur et le moi en tant que manager, celui-ci a le sentiment de «jouer sa peau » pour reprendre une expression utilisée dans le langage courant, mais qui, ici, nous semble bien décrire de quoi il s'agit. Celui qui a le sentiment de «jouer sa peau» ne serait-il pas dans une sorte de "fusion » entre le jeu et la réalité ? Les conséquences sur le réel semblent acceptées par François sans pour autant le «détruire» personnellement ou professionnellement.

\section{Une arène de jugement}

41 Mais est-ce si fantasmatique que cela de «jouer sa peau "? Quelle est la prégnance de la menace de l'évaluation et du risque de relégation dans un environnement compétitif où il y a des gagnants et des perdants ? Quelle possibilité est laissée au joueur de refuser de jouer quand le jeu s'inscrit dans une formation obligatoire pour son évolution de carrière et qu'il se déroule sous le regard des pairs et des formateurs?
[...] il y a plein de choses sur lesquelles les gens peuvent se dire: "là-dessus je pourrais être jugé » et on va se dire «si jamais je me foire à être un bon chef de bord on va se dire que je ne suis pas un bon manager ", on ne sait pas à quel point on est jugé, alors que c'est bidon, c'est difficile de sortir de ça, si on n'est pas dans un mode jeu, on va se sentir jugé, si le chef lui envoie des phrases assassines : « tu n'y es pas encore arrivé », il va le prendre pour lui et l'autre peut se dire : « il pense que je suis nul ", je pense que ça peut très vite dériver.

Le risque de ce genre de pratique est de mettre les managers en visibilité dans une "arène de jugement" (Dodier, 1993; Jobert, 2014) où les traits de caractère ressortiraient plus que les savoir-faire. Dans cette mise en visibilité, il y a des effets de révélation: pour soi et pour les autres. En plus des caractéristiques personnelles soulignées, François va aussi relever l'importance du groupe contenant et soutenant dans lequel se déroule le jeu : 
[...] il y a une dimension play dans l'interaction, etc. pour le coup ça dépend vraiment des groupes, ça dépend des personnes avec qui on joue, etc. après les personnes qui ont l'habitude de jouer, et de se mettre dans ce genre de situation, ils seront plus à l'aise, et seront plus dans du play, et tourneront autour des règles, etc. par contre si on est avec des gens qui ont l'habitude de suivre des règles, s'ils vont rentrer dans le game, et justement la discussion que l'on a eue, on était dans le play, on était à se challenger un peu, il y avait un côté ludique à essayer de trouver la bonne stratégie [...] anecdote: «lorsque l'on me demande depuis combien de temps je fais de la programmation, je réponds 30 ans et ça fait sourire», puis il ajoute « et c'est vrai ». Il nous explique qu'il a programmé son premier jeu à 8 ans. Un jour il a demandé à son père un jeu vidéo et celui-ci lui a offert son premier ordinateur en l'encourageant à programmer son propre jeu. Le jeu a toujours été ainsi lié, selon François, à la valeur travail. François est issu d'un milieu modeste explique-t-il (mère infirmière et père mécanicien), la valeur «travail » était très importante. Il raconte comment sa mère avant son entrée en primaire lui apprit à lire l'heure avec un biscuit coupé en quatre pour les quarts d'heure. L'environnement de François dans son enfance était propice à l'apprentissage par le jeu. Les jeux de plateaux ou de cartes avaient aussi leur place en tant que loisir les week-ends pluvieux et les soirées en famille.

Aujourd'hui, François travaille sur l'intelligence artificielle et il est aussi joueur de jeux de plateaux et de jeux de rôle dans sa vie. Il a transféré des compétences acquises dans une activité dans une autre activité, mais aussi au sein de son équipe avec qui il a développé des activités « ludiques ». Il est important ici de préciser que le ludique est relatif au jeu.

- quizz informels lors de l'intégration des nouveaux membres de l'équipe pour que chacun apprenne à mieux se connaître ; 
- post-it affichés sur un tableau avec des couleurs distinctes en fonction de l'humeur de l'équipe, afin de donner à François des indicateurs sur le climat émotionnel.

Il nous semble que François possède les trois caractéristiques inhérentes à l'attitude ludique : l'illusion, la duplicité ainsi que la capacité à être et à agir dans l'incertitude (Henriot, 1969). Il explique « le jeu ne se déroule pas comme prévu donc cela ne sert à rien de trop planifier les choses». Son exemple impliquerait-il que seules des personnes ayant développé une attitude ludique dans l'enfance ou dans la sphère privée seraient en capacité d'avoir une attitude ludique en formation, au travail ?

Nous avons observé quatre autres managers également joueurs dans la vie qui, dans SimLead, n'ont pas développé de «trucs malins» pour reprendre l'expression de François. Lorsque nous leur donnons cet exemple, ils expliquent « qu'ils n'auraient pas pensé que ce soit possible ». Qu'est-ce qui amène des managers à se conformer aux attendus dans SimLead ? Qu'en est-il des autres managers? Nous précisons que François est une exception sur les 44 managers, la plupart des managers ont vécu le «jeu» comme un exercice, comme une journée de travail "sous pression». Ceci nous a amenés à nous interroger sur les empêchements à l'attitude ludique dans de tels dispositifs.

\section{Normalisation et conformations aux directives}

\section{Un métier très cadré}

51 L'environnement du jeu lui-même est très contraint, le play n'est pas favorisé dans SimLead. Cet environnement conduit ainsi à l'exécution de la tâche, mais pas à son questionnement ni à sa réorganisation. Le pilotage d'hélicoptère est une manœuvre qui laisse peu de place à l'initiative et à la négociation des règles, bien que certains managers aient négocié leur place dans l'hélicoptère et le temps alloué à la mission, par exemple. Ils vont cependant éprouver la pénibilité et l'inconfort d'une situation très contrainte. Ceci peut être rassurant pour certains, cantonnés dans l'exécution, sans avoir à réfléchir ni à prendre des risques.

Les managers ont un code de conduite, et dans le jeu, espace d'expérimentation, le réel reste collé au prescrit. Il n'y a pas de jeu au niveau des décisions ou des règles. Le jeu, qui les met dans une situation virtuelle, devrait permettre d'accroître la distance entre soi et l'artefact pour expérimenter et négocier des règles. Nous nous sommes demandés si la culture d'entreprise, ici militaire, n'était pas mise en avant pour justifier cette tendance à la soumission comme un mécanisme de défense de par la sécurité qu'elle procure (Amado, 1998). Au lieu de favoriser les processus d'individuation, l'usage d'un tel dispositif semble ainsi instrumentaliser une cohésion organisationnelle et une idéologie managériale.

Olivier Mauco (2008) explique que les jeux sérieux diffusent des messages majoritairement politiques et les assimile à des "médias politiques». Le message politisé est ainsi contenu dans le dispositif au travers « des règles du jeu, des cadres de l'expérience, du système d'action individuel proposé et des modalités de résolution des problèmes » (Mauco, 2009, p. 17). Les scénarii développés par les concepteurs, avec les décisions que le joueur peut ou ne pas prendre, permettraient aux concepteurs de faire passer des messages implicites. "L'appellation même de serious game permet de dépolitiser ce média, le présentant comme un nouvel outil technique d'information et 
non comme un instrument idéologique" (Mauco, 2008, p. 79). Sous la métaphore ludique, nous retrouvons ainsi le contrôle et l'instrumentation prescrite d'une idéologie.

A cet environnement contraint s'ajoute l'environnement psychosocial actuel très contraint ainsi que le métier d'ingénieur où l'application des procédures prime (malgré l'innovation qui est au cœur du métier). Quelle place pourrait laisser un ingénieur à l'« incertitude » et à l'« illusion » nécessaires à l'attitude ludique que pourrait adopter un joueur? Cependant, nous retrouvons les mêmes contraintes dans les fonctions supports où Martine nous rappelle que son métier de responsable finance est très cadré.

Il faut comprendre que dans la direction financière, on est complètement régi par des règles; on n'est ni dans l'innovation ni dans la créativité ; on est très borné. Par contre, ça évolue très vite ; on doit se tenir au courant.

Puis elle ajoute au cours de l'entretien «nous sommes en contrôle et nous devons reporter et être réalistes, fiables dans ce que l'on reporte vers notre direction ».

Martine fait un lien entre la simulation et l'activité réelle de travail lors des « changements de cap » qui renvoient à la clôture trimestrielle, ce qui pour elle est source de tensions :

Les changements de cap, tout le temps: on a de gros exercices budgétaires où on nous donne des hypothèses avec travail à la clé avec des hypothèses qui peuvent être changées par le management à une semaine de la finalisation de cette revue pour présentation; et là, c'est la catastrophe ; le travail dans l'urgence, 15 heures de boulot par jour. Et, à un certain moment, on a envie de tout laisser tomber, de partir en courant, de se dire je n'y arriverai jamais avec un niveau de stress énorme; et chaque fois que ça arrive, on espère que la fois d'après on le gèrera mieux. Ces changements de cap, c'est un écho. [...] En fait, le vécu vient parasiter un exercice qui n'avait pas vocation à mettre un stress énorme. L'expérience que j'ai au travail a forcément influé sur la façon dont j'ai pris le jeu.

57 L'expérience personnelle et professionnelle, c'est-à-dire l'éprouvé, pourrait être un frein à l'attitude ludique si les conditions dans lesquelles se déroule le jeu sont trop similaires au réel. Le mot "expérience» vient étymologiquement du terme grec: épreuve. Jobert propose une définition de l'expérience :

L'expérience c'est ce qui s'est constitué, au fil du temps, individuellement et collectivement, dans l'intimité des personnes, dans leur corps, leur intelligence, leur imaginaire, leur sensibilité, dans la confrontation quotidienne avec le réel et la nécessité de résoudre des problèmes de toute nature (Jobert, 1991, p. 75).

Elle peut-être une contrainte, mais est aussi une ressource qui peut être mobilisée dans de nouvelles situations.

Ici, les caractéristiques citées précédemment ne trouvent pas leur place. L'illusion, l'incertitude et la duplicité sont empêchées par une expérience professionnelle et un dispositif de formation qui y fait écho. Nous n'avons pas réalisé de récit de vie avec Martine, ce qui nous aurait certainement permis d'approfondir les raisons de son vécu au regard de sa trajectoire sociale et individuelle. Nous nous appuierons sur l'expérience de Martine pour analyser les contraintes psychiques, constituant des empêchements à l'attitude ludique. 


\section{Les contraintes psychiques}

\section{d'évaluation viennent écraser celle du jeu.}

Pour moi, à aucun moment je ne l'ai perçu comme un jeu, mais plutôt comme un exercice qui allait faire ressortir quelque chose. [Elle parle] d'analyse de comportements [et elle en dit] en fait, je ne l'ai pas bien vécu du tout: grosse remise en question le jour même; je me suis sentie très mal.

Martine, comme la plupart des managers, s'est sentie évaluée en tant que «manager » dans une formation managériale et sous le regard d'autrui. A cette évaluation extérieure s'ajoute son auto-évaluation. Le dispositif de formation avec son discours idéologique sur le bon «manager", le «leader et l'équipe performante", met en lumière, au travers de SimLead, un idéal. Cet idéal met en exergue les «manques », les «failles » au regard de cette idéologie prescrite, ce qui provoque de la souffrance et une adhésion plus spontanée aux ordres pour se rapprocher de cet idéal.

Il existe une estimation subjective de ce que la technologie va reconnaître et/ou mettre en valeur dans la contribution du sujet: en termes d'expériences, de qualifications, d'aptitudes, mais aussi d'utilité et de reconnaissance sociale et professionnelle (Bobillier-Chaumon, 2013). SimLead s'éloigne alors du développement des compétences managériales, du faire pour se déplacer sur l'être.

Pour Martine, l'expérience a fait ressortir :

Mes points faibles que je connaissais déjà : l'inconnu qui fait peur, vouloir tout contrôler à tout prix et ne pas laisser d'espace aux autres parce que tant que je ne suis pas en maîtrise ça ne va pas.

64 Nous sommes confrontés à une double contrainte entre la prescription du jeu non souhaité et le ressenti évaluatif. L'activité d'évaluation, qui renvoie à l'organisation, empêche le jeu de se déployer et certains, comme Martine, s'enferment dans le bien faire, le travail prescrit. L'incertitude est vécue ici comme dangereuse, le non-contrôle d'un comportement est exclu.

La fragilisation du rôle du manager dans le processus de travail au cours même de la simulation est soulevée. Ceux-ci disent que cet instrument est «un révélateur de personnalité ». En dehors de SimLead, nous rappelons qu'ils ont déjà été soumis le jour précédent à un questionnaire de personnalité (MBTI), ce qui accentue ce sentiment de dévoilement du Moi. Le fait de mettre les personnes dans un environnement dynamique amplifie cet effet révélateur: "ça permet de voir comment les gens réagissent sous pression», nous explique un formateur. Nous précisons que la signification du mot "serious» en anglais signifie « sérieux", mais aussi "grave", «dangereux». Martine a mal vécu cette expérience qui a été violente à vivre symboliquement.

\section{Une expérience qui « secoue »}

Certains managers vont refouler leurs émotions en projetant sur les autres ce qu'ils ont ressenti : «ils ont stressé, pas moi », « certains se sont sentis évalués ». La vulnérabilité que le dispositif réactive face au groupe de pairs, à l'instructeur et aux formateurs dépasse le cadre même de la formation. Les managers ne sont pas en "soin ", mais en formation et les formateurs ne sont pas formés pour faire face à la mise en visibilité de 
cette vulnérabilité. Ces derniers pourraient mettre au travail dans le débriefing la réaction du groupe qui souvent va jusqu'à l'exclusion de celui qui a un comportement déviant. Le " jouer sa peau » n'est pas imaginaire, il est bien réel. Les managers vivent l'expérience de SimLead comme un «exercice » d'évaluation qui met à l'épreuve leurs compétences managériales.

Martine partage ce sentiment qu'elle a eu : «je n'ai pas été à la hauteur ». A la hauteur de quoi ? D'après ses dires, c'est elle qui est à l'origine du problème, à la peur et la colère succèdent l'auto-culpabilisation, la honte et la perte d'une identité "d'homme capable» tel que défini par Ricœur dans son livre «de l'homme faillible à l'homme capable » et qui décline le «je peux » sous différentes modalités : «je peux dire», «je peux faire», «je peux raconter» et «je peux m'imputer la responsabilité de mes actes » (Almudever, Michaëlis, Aeschlimann \& Cazals-Ferré, 2012). Cette figure «d'homme capable » est particulièrement intéressante lorsqu'il s'agit d'une femme dans un monde d'hommes. Martine a demandé la formation, car elle a à cœur d'« être un bon manager ».

Lorsqu'elle se retrouve chef de bord, elle dit : «pour le $1^{\mathrm{er}}$ exercice, j'ai été mise leader. Pourquoi? J'avais une bonne tête de gagnante, je ne sais pas, j'avais peut-être laissé apparaître une façon d'être ». Les projections de Martine ont pris le dessus avant même le début du jeu. Les places s'attribuent en fonction de l'emplacement des managers lorsqu'ils rentrent dans la salle. Ils prennent place sur les chaises et s'installent. S'ils ne font pas attention aux étiquettes qui mentionnent le rôle de chef de bord et de pilote ou à la manette de jeu (qui est aussi un indicateur), ils ne savent pas qu'ils viennent de s'attribuer un rôle. Nous avons observé que les plus aguerris, joueurs par ailleurs, vont directement vers la manette de jeu, car pour eux « jouer, c'est piloter l'hélicoptère ».

Les projections de Martine l'accompagnent tout le long du jeu. Ainsi, au moment où, lors d'une mission, un autre leader a été désigné, elle s'est interrogée sur sa capacité à diriger.

Le changement de cap dans l'acte 2 ( $2^{\mathrm{e}}$ exercice), je ne l'ai pas mal vécu, enfin je l'ai mal vécu entre guillemets, car je n'ai pas compris la consigne, il y a eu une période de flottement où c'est finalement Eric (le navigateur) qui m'a dit «mais c'est elle qui a pris le lead », en parlant de Sandrine (le chef de bord de l'autre hélicoptère) ; et là je me suis dit «j'étais pas assez bien et du coup on me l'a enlevé ». [...]. La réaction était toujours très personnelle; de se dire «mince je n'étais pas à la hauteur, c'est le ressenti du moment $»$.

70 Le leader de la mission VIP désigné est aussi une femme, ce qui a peut-être favorisé ce sentiment de ne pas avoir été à la hauteur, qui plus est parce que cette femme était aussi chef de bord dans l'autre hélicoptère au sein de la même équipe. Ce que Martine ne sait pas, c'est que le leader de cette dernière mission est désigné en fonction des positions des hélicoptères et non des prestations des managers en tant que chef de bord. Si l'hélicoptère est proche de la dernière zone de sauvetage, le chef de bord est choisi pour guider les quatre autres hélicoptères.

\section{La place des jeux de rôle dans le dévoilement de soi}

71 Les jeux de rôle utilisés en formation permettent de prendre des décisions et d'en explorer les conséquences. Dans SimLead, les managers jouent un rôle. Nous faisons l'hypothèse que le jeu de rôle engage la personne différemment. 

(1973), il existe deux aspects à différencier et à la fois intimement liés. Ce sont l'aspect social (conventions sociales, attentes de rôles, etc.) et l'aspect individuel, qui est une sorte de réappropriation par chacun (Moreno, 1965). Dans l'aspect social, on retrouve les normes de la conduite et de l'apparence que le groupe social y associe (Goffman, 1973). La spontanéité échappe aux managers et dévoile un moi authentique pour certains. D'autres dissimulent leurs émotions et sentiments afin de répondre à des contraintes sociales. L'approche goffmanienne souligne l'importance des coulisses qui permettent collectivement de prendre des décisions. Ces coulisses, qui pourraient être des moments de délibérations ou de débriefings, ne sont pas très présentes, la prise de distance est ainsi écrasée par la précipitation. Si une rupture se produit dans l'interaction, elle peut détruire l'image de soi autour de laquelle la personnalité de l'acteur s'était constituée, ici celle du manager.

Le vécu de Martine illustre bien notre propos. Le jeu de rôle fait émerger l'être et cette image du Moi qui surgit devant les autres se veut honorable et digne. La liaison entre un monde intérieur et un monde extérieur doit se faire. Cette représentation quotidienne, dont une des ressources est constituée par les normes internalisées sur le rôle à tenir, fait partie de l'activité du manager. Elle ouvre des potentialités du côté du réel et du sujet et est source de créativité si l'illusion est maintenue.

\section{Prescription et proscription des émotions}

Brunel $(2006,2008)$ explique que ce travail sur soi, l'usage de cette réflexivité, repose sur l'ambiguïté du dévoilement d'un moi authentique et l'apprentissage de codes relationnels et comportementaux socialement utiles qui contribuent à l'exercice du pouvoir organisationnel. La question se pose alors de savoir dans quelle mesure ces pratiques managériales sur le savoir-être participent à un mouvement de dichotomisation de la société : il y aurait ceux qui auraient le potentiel et les autres (Brunel \& Cultiaux, 2002). Le vulnérable est toujours l'autre, la vulnérabilité humaine, en ce sens que nous sommes tous vulnérables, s'efface au profit d'une vulnérabilité sociale.

Cependant, nous avons aussi évoqué que dans la situation de Martine, la fonction soutenante du groupe a été manquante : Martine n'a pas eu le sentiment de faire partie d'une équipe. Le dispositif positionne des pairs comme membre d'une même équipe, ce qui vient questionner la confiance favorisant la coopération pour faire équipe (Lhuilier, 2014). L'objectif même de SimLead est donc à réinterroger lorsque des pairs sont amenés à travailler en équipe. Qu'est-ce qui fait qu'à un moment donné Martine a eu le sentiment de ne pas être à la hauteur ? Et à la hauteur de quoi ? Qu'a-t-elle intériorisé pour avoir ce sentiment? Peut-on parler de jeu?

Le groupe ne constitue pas une ressource pour gérer l'émotion, ce qui empêche le jeu, l'expérimentation et la créativité de se mettre en place. Les tensions émotionnelles ne sont pas abordées, ce qui ne permet pas de rechercher de nouveaux modes d'organisation du travail d'équipe ou de coopération qui allègent le poids des contraintes. Le sujet doit ainsi assumer seul les difficultés, les risques et les échecs des actions, et surtout travailler dans l'incertitude constante d'être en adéquation avec les attentes (souvent implicites) de l'organisation. 
77 Sans un cadre d'expression de ses résonances émotionnelles, le groupe de travail ne peut pas réguler les contraintes de travail et avoir un rôle de médiateur entre l'individu et la situation de travail (Lhuilier, 2006). La charge cognitive (charge de travail, intensification, etc.) et la charge affective (inconfort émotionnel, émotions prescrites et empêchées) semblent déterminantes dans la façon dont l'artefact technologique est appréhendé et utilisé (Bobillier Chaumon, 2013). Les représentations sont atténuées si le groupe est une ressource pour jouer un rôle d'intercalaire qui protège face aux exigences de l'organisation, les autres deviennent alors une ressource pour travailler.

\section{Conclusion}

78 Dans de tels dispositifs, il nous semble essentiel de nous interroger sur le rôle et les attentes des concepteurs. En effet, en décontextualisant l'apprentissage, ici des managers qui pilotent un hélicoptère, la question de l'usage ne peut pas être abordée de la même façon qu'avec des pilotes d'hélicoptères formés avec le simulateur EDITH. Qu'est-ce qui a pu motiver la décision de mettre des managers dans des conditions dynamiques qui les renvoient à leurs situations de travail ? L'objectif est-il de les amener à transformer leur situation de travail ou à s'y adapter? Voulait-on simplement rendre naïvement une formation plus « amusante »?

79 Nous supposons que l'intitulé de serious game a été une opportunité de par l'effet de mode autour de ces logiciels. Il s'agirait ici plutôt de la gamification d'un simulateur qui en soi n'est pas concluante pour pouvoir parler de «jeu». Cela pose la question de la gamification: la transposition analogique d'un simulateur suffit-elle pour parler de gamification? Le contexte dans lequel se déroule le jeu est central. Le côté sérieux est préservé au travers du détournement de l'outil militaire, source de crédibilité et en rapport avec la culture de l'entreprise.

80 Notre article démontre que la marge du jeu est inhérente à l'attitude subjective des personnes qui peuvent rester en marge du jeu (ou pas) particulièrement avec des jeux sérieux. Cette attitude est profondément liée à la trajectoire personnelle et professionnelle qui est un enchevêtrement entre histoire de vie, éducation, personnalité, contexte et situation ludique. Les différentes perceptions vis-à-vis d'un dispositif présenté comme ludique éclairent toute la subjectivité autour de la marge du jeu. Il ne s'agit donc pas de «parler de jeu » en général, mais de s’intéresser au "vécu $\mathrm{du}$ jeu » de chacun. Ces diverses perceptions au cours de l'usage font que l'on peut le ressentir comme un jeu ou comme un exercice contraint et culpabilisant.

81 Nous sommes confrontés à une double activité : une activité d'évaluation sous son propre regard, celui d'autrui, et une activité de jeu, du moins présentée comme telle. L'activité d'évaluation, qui renvoie à l'organisation, peut empêcher le jeu de se déployer. Un enjeu majeur ressort de SimLead: l'évaluation des compétences émotionnelles en tant que manager. L'exemple du mal-être de Martine est un bel exemple de cette conformation à laquelle elle résiste, ce qui amène la question suivante: conformer est-ce former? Cette éventuelle dérive d'une évaluation des compétences vers une évaluation de la personne dans une totale occultation du travail et de son cadre ne favorise pas la prise en charge émotionnelle comme un objet du travail (Lhuilier, 2006). La question du dévoilement du Moi est un enjeu très personnel de la relation du Moi au jeu (ou je). Est-il possible de s'en dégager pour maintenir l'illusion et « jouer »? 
, nous pourrions nous interroger sur cette dimension « jeu»: n'est-elle pas une manière euphémisante pour former à quelque chose de mal défini : le management ? Peut-on former au management? Il nous semble essentiel dans les dispositifs de formation de s'interroger sur l'objet même de la formation avant de questionner les modalités pédagogiques. Qu'est-il prévu d'apprendre ? Et en quoi le passage par le jeu peut y aider?

\section{BIBLIOGRAPHIE}

ALMUDEVER, B. (2007), Créativité individuelle et collective au travail : enjeux de personnalisation et de changement social, Mémoire HDR, Université Toulouse-Le Mirail.

ALMUDEVER, B., MICHAELIS, N., AESCHLIMANN, M. P., et CAZALS-FERRE, M. P. (2012), Le pouvoir d'agir à l'épreuve de la souffrance au travail : émotions, recherche et construction de sens. Psychologie du Travail et des Organisations, vol. 18, t. 1, pp. 81-101.

ALVAREZ, J. (2007), Du jeu vidéo au serious game : approches culturelle, pragmatique et formelle, thèse de doctorat, Toulouse 2.

ALVAREZ, J., et DJAOUTI, D. (2010), Introduction au Serious Game, Paris, Questions théoriques.

ALVAREZ, J., LIBESSART, A., et HAUDEGOND, S. (2014), « Le "jeu non sérieux", une activité improductive? ", Interfaces Numériques, vol. 3, t. 3, pp. 391-408.

ALVAREZ, J., (2015), Enrichissement d'un modèle évaluatif pour assurer une formation avec le jeu comme médiation, Journée AIM Serious Games et Co-design, GEM.

AMADO, G. (1998), « Cohésion organisationnelle et illusion collective », Revue française de gestion, $\mathrm{n}^{\circ} 69, \mathrm{pp} .37-43$.

BOBILLIER-CHAUMON, M.E (2013), Conditions d'usage et facteurs d'acceptation des technologies: Questions et perspectives pour la psychologie du travail, Mémoire HDR, Université Pierre MendèsFrance, Université Lyon 2.

BROUGERE, G. (2005), Jouer/Apprendre, Paris, Education.

BROUGERE, G. (2012), « Le jeu peut-il être sérieux ? Revisiter Jouer/Apprendre en temps de serious game ». Australian Journal of French Studies, vol. 49, t. 2, pp. 117-129.

BROUGERE, G. (2013), « Jacques Henriot et les sciences du jeu ou la pensée de Villetaneuse ». Sciences du jeu, $\mathrm{n}^{\circ} 1$, http://sdj.revues.org/202 ; DOI: 10.4000/sdj.202

BRUNEL, V. (2006), « Pratiques réflexives et régulation organisationnelle », Communication et organisation, $\mathrm{n}^{\circ} 28, \mathrm{pp} .17-31$.

BRUNEL, V., et CULTIAUX, J. (2002), « Le développement de l'individu managérial », Actes du XIIIè congrès de l'association internationale des psychologues du travail et de la formation.

BRUNEL, V. (2008), Les managers de l'âme : le développement personnel en entreprise, nouvelle pratique de pouvoir ? Paris, La Découverte.

CAILLOIS, R. (1991), Les jeux et les hommes : le masque et le vertige, Paris, Gallimard. 
CORTAZAR, J. (2013), Clases de Literatura, Berkeley, 1980, Buenos Aires, Alfaguara.

CURIE, J., et HAJJAR, V. (1987), « Vie de travail. Vie hors travail : la vie en temps partagé », in C.Lévy-Leboyer \& J. C. Spérandio (dir.), Traité de psychologie du travail, Paris, PUF, pp. 35-55.

CURIE, J., et DUPUY, R. (1994), « Acteurs en organisations ou l'interconstruction des milieux de vie » in C. Louche (dir.) Individus et organisations, Neuchâtel, Delachaux \& Niestlé, pp. 53-80.

DODIER, N. (1993), « Les arènes des habiletés techniques. Les objets dans l'action », Raisons pratiques, $\mathrm{n}^{\circ} 4$, pp. 115-139.

GOFFMAN, E. (1973), La mise en scène de la vie quotidienne. Tome 1. La présentation de soi, Paris, Minuit.

HENRIOT, J. (1969), Le Jeu, Paris, PUF.

HENRIOT, J. (1989), Sous couleur de jouer : la métaphore ludique, Paris, José Corti.

HUIZINGA, J. (1988), Homo ludens, essai sur la fonction sociale du jeu, Paris, Gallimard.

JOBERT, G. (1991), « La place de l'expérience dans les entreprises », in B. Courtois, G. Pibeau et al. (dir.). La formation expérientielle des adultes, Paris, La documentation française, pp. 75-82.

JOBERT, G. (2014), Exister au travail. Les hommes du nucléaire, Toulouse, Eres

JUUL, J. (2003), « The game, the player, the world: looking for a heart of gameness ", in C. Marinka \& R. Joost (Eds.), Level Up Conference Proceedings: Proceedings of the 2003 Digital Games Research Association Conference, Utrecht, University of Utrecht, pp. 30-45. https:// www.jesperjuul.net/text/gameplayerworld/

LAVIGNE, M. (2014), Les faiblesses ludiques et pédagogiques des serious games. http://upstlse.academia.edu/MichelLavigne

LAVIGNE, M. (2016), «Jeu et non jeu dans les serious games », Sciences du jeu, n 5, http:// sdj.revues.org/648 ; DOI: 10.4000/sdj.648

LELARDEUX, C., PANZOLI, D., ALVAREZ, J., GALAUP M., et LAGARRIGUE, P. (2012), Serious Game, Simulateur, Serious Play : état de l'art pour la formation en santé, e-virtuoses/SEGAMED. http:// www.ludoscience.com/files/ressources/SimulatorSG.pdf

LELARDEUX, C., ALVAREZ, J., MONTAUT, T., GALAUP, M., \& LAGARRIGUE, P. (2012), « Healthcare Games and the Metaphoric Approach », in S. Arnab, I. Dunwell \& K. Debattista (eds), Serious Games for Healthcare: Applications and Implications, IGI Global, pp. 23-49.

LE LAY, S. (2013), « Des pratiques ouvrières ludiques aux dispositifs managériaux ludistes : vers une instrumentalisation du jeu dans le travail », in La nouvelle revue du travail, $\mathrm{n}^{\circ} 2$. http:// nrt.revues.org/702

LHUILIER, D. (2006), « Compétences émotionnelles : de la proscription à la prescription des émotions au travail », Psychologie du travail et des organisations, vol. 12, t. 2, pp. 91-103.

LHUILIER, D. (2013), « Introduction à la psychosociologie du travail », Nouvelle revue de psychosociologie, $\mathrm{n}^{\circ} 15, \mathrm{pp}$. 11-30.

LHUILIER, D. (2014). « Construire le "faire ensemble” », Revue Santé Mentale, n 186, pp. 20-27.

MAECANO, B. (2008), « Juegos serios y entrenamiento en la sociedad digital », Teoría de la Educación. Educación y Cultura en la Sociedad de la Información, vol. 9, t. 3, pp. 96-106 http:// campus.usal.es/ teoriaeducacion/rev_numero_09_03/MONOGRAFICO_VIDEOJUEGOS.pdf 
MARTIN, L. (2015). « Le serious gaming, un outil de formation des cadres : une réflexion sur la transitionnalité », Education permanente, $\mathrm{n}^{\circ} 202$, pp. 81-90.

MENDEL, G. (1998), L'acte est une aventure ; du sujet métaphysique au sujet de l'acte pouvoir, Paris, La Découverte.

MAUCO, O. (2008), « Les serious games, entreprise d'auto-légitimation », MédiaMorphoses, $\mathrm{n}^{\circ} 22$, pp. $79-84$.

MAUCO, O. (2009), «Les serious games : état des lieux de jeux vidéo institutionnalisés », Séminaire DEL.

MORENO, J. L. (1965), Psychothérapie de groupe et psychodrame, Paris, PUF.

PASTRE, P. (2005), « La simulation en formation professionnelle », in P. Pastré (dir.) Apprendre par la simulation. De l'analyse du travail aux apprentissages professionnels, Toulouse, Octarès. pp. 7-16.

POMIAN, J. (2009), « Serious games : apprendre en jouant ?» Quaderni, n 69, p. 121-126.

RINAUDO, J.L. (2011), Négatif, transitionnel, groupe. TIC, éducation et psychanalyse, Paris, L'Harmattan.

SIMON, L. (2005), « Du gamesman aux gamers : le jeu comme dynamique émergente des organisations contemporaines?» Gestion, vol. 30, t. 4, pp. 34-42.

TRICLOT, M. (2011), Philosophie des jeux vidéo, Paris, La découverte.

WINNICOTT, D.W. (1970), « Vivre créativement », In Winnicott, D.W., Conversations ordinaires, Paris, Gallimard. pp. 54-77.

WINNICOTT, D.W. (1971), Jeu et réalité : l'espace potentiel, Paris, Gallimard.

\section{RÉSUMÉS}

Cet article pose la question de la frontière entretenue entre le jeu et le travail avec un simulateur appelé serious game par les concepteurs. Nous nous sommes intéressés à un simulateur d'hélicoptère utilisé pour former 350 managers par an. Cette recherche qualitative dans le cadre théorique et méthodologique de la psychosociologie du travail vise à comprendre la perception que les professionnels, ici des managers, ont du dispositif et de son environnement ainsi que la façon dont ils se comportent et agissent. Nous avons rencontré 44 managers volontaires du même grand groupe industriel ayant tous suivis cette formation managériale. Les données qualitatives, par essence complexes et ouvertes, ont été collectées suivant trois modalités: observations in situ pendant l'activité dans le monde virtuel, entretiens individuels avant et un mois après l'expérimentation sur le lieu de travail. Nous interrogeons ici les frontières du jeu quand il devient une pratique prescrite dans des dispositifs de formations managériaux.

This article explores the boundary between the world of simulation games (referred to as "serious games »), and work. Our interest was in examining a helicopter simulator integrated game used to train 350 managers per year. This qualitative research, based on the theoretical and methodological framework of the psychosociology of work, aims at understanding the perception which professionals, namely managers, have, of the device and its environment, as well as the way they behave and act. We met 44 voluntary managers of a big industrial group, all having completed this managerial training course. The qualitative data, in essence, complex and open, was collected via three methods: situational observations during the activity in the virtual world, 
individual interviews in the workplace before and one month after the experiment. We question here the borders of the game when it becomes a practice prescribed in managerial trainings.

INDEX

Mots-clés : jeu, exercice, serious game, formation, attitude ludique

Keywords : game, exercise, serious game, training, playful attitude

\section{AUTEUR}

LYDIA MARTIN

CRTD - CNAM 